\title{
研究通讯
}

\section{Sasaki 流形的拟脐超曲面}

设 $\widetilde{M}^{2 n+1}$ 是结构张量组为 $\left(F_{A}^{B}, G_{A B}, F^{A}\right)$ 的 Sasaki 流形, $M^{2 n}$ 是等距浸人在 $\widetilde{M}^{2 n+1}$ 中 的超曲面. $\widetilde{M}^{2 n+1}$ 的结构张量组在 $M^{2 n}$ 上的 诱导结构为 $\left(f_{a}^{b}, g_{a b}, u^{a}, v^{a}, \lambda\right)^{[1]}, N^{A}$ 为 $M^{2 n}$ 在 $\widetilde{M}^{2 n+1}$ 中的单位法向量, 其中 $\lambda$ 是 $\widetilde{M}^{2 n+1}$ 中 的结构向量 $F$ 与 $M^{2 n}$ 的法向量 $N$ 的夹角的余 弦, 即 $\lambda=\cos \langle F, N\rangle$.

设 $M^{2 n}$ 为基本元为 $v^{a}$ 的拟脐超曲面 ${ }^{[2]}$, 即它的第二基本形式满足: $h_{a b}=p g_{a b}+q v_{a} v_{b}$, 若 $q=0$, 则 $M^{p n}$ 是全脐的, 特别若再有 $p=$ const. $\neq 0$, 则称为特征全脐超曲面; 若 $p=0$, 则 $M^{2 n}$ 是柱形的; 若 $p=q=0$, 则 $M^{2 n}$ 是全 侧地的.

利用 $M^{2 n}$ 上的诱导结构中的向量场 $u^{a}$ 和 $v^{a}$, 构作了 $M^{2 n}$ 上的两个新向量场: $\mathscr{L}_{v} u=$ $[u, v]$ 和 $v^{b} \nabla_{b} u^{c}-v^{c} \nabla_{b} u^{b}$ (其中 $\mathscr{L}_{v}$ 是关于 $v$ 的 Lie 导数算子), 分别求出了它们的散度, 并利 用 Green 定理得出了 $M^{2 n}$ 的两个积分公式, 利用这些积分公式,证明了

定理 1 设 $M^{2 n}$ 是 Sasaki 流形 $\widetilde{M}^{2 n+1}$ 的紧 致可定向的拟脐超曲面, 即 $h_{a b}=p g_{a b}+q v_{a} v_{b}$, 其基本元 $v_{a}$ 为在 $M^{2 n}$ 诱导结构向量, 且 $q=$ const.. 若 $\cos ^{2}\langle F, N\rangle \leqslant \frac{1}{2 n+3}$ 或 $\cos ^{2}\langle F$, $N\rangle \geqslant \frac{1}{2 n+3}$, 则有下列形表之一发生:

(1) $M^{2 n}$ 是全脐的; (2) $\tilde{M}^{2 n+1}$ 的结构向量 $F$ 与
$M^{2 n}$ 的法向量交定角 $\left(\cos ^{2}\langle F, N\rangle=\frac{1}{2 n+3}\right)$.

定理 2 设 $M^{2 n}(n>1)$ 是 Sasaki 空间形 式 $\widetilde{M}^{2 n+1}$ 的紧致可定向超曲面, 即 $h_{a b}=p g_{a b}+$ $q v_{a} v_{b}$, 其中基本元是在 $M^{2 n}$ 上的诱导结构向 量 $v_{a}$ ，若 $p \geqslant 0, \lambda^{2} q \geqslant p$ 或 $p \leqslant 0, \lambda^{2} q \leqslant p$, 则有 下列情形之一发生：(1) $M^{2 n}$ 是全测地的;

(2) $F$ 切于 $M^{2 n}$, 且 $M^{2 n}$ 是柱形的.

利用 Obata 关于 Riemann 流形等距于 球面的著名定理, 我们研究了完备特征全脐 超曲面, 得出了

定理 3 设 $M^{2 n}$ 是 Sasaki 流形 $\widetilde{M}^{2 n+1}$ 的 完备特征全脐超曲面, 且结构向量 $F^{A}$ 的法 部有变动长度, 则 $M^{2 n}$ 等距于 $E^{2 n+1}$ 中半径为 $2 n / \sqrt{H^{2}+4 n^{2}}$ 的超球面.

\section{参 考 文 献}

1 Yano K, Ki U H. Infinitesimel variations typersurfdace of a Sasakian mannifold. Tensor N S, 1979, 33: $1 \sim 10$

2 Chen B Y, Yano K. Hypersuface of a conformally flat space. Tensor N S, 1972, 26: 318 322

3 Yano K. Integral Formulas in Riemann Geometry. New York: Marœl Dekker Inc, 1970

\section{罗崇善}

(四川师范大学数学系, 成都 610066) 